\title{
Schweizerische Gesellschaft für Pathologie
}

Pierre-André Diener

Sekretär der SGPath

Dr. med. Pierre-André Diener Institut für Pathologie Kantonsspital St. Gallen CH-9007 St. Gallen

p.a.diener@kssg.ch

www.sgpath.ch

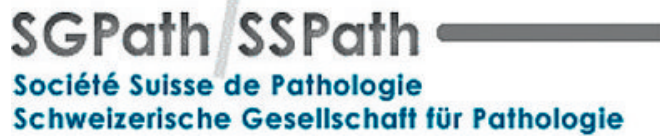

\section{Was ist Pathologie}

Die Pathologie handelt vom Verstehen der Krankheiten, ihren Ursachen, den Prozessen im Krankheitsverlauf sowie deren morphologischen und molekularbiologischen Veränderungen.

Es ist in der Öffentlichkeit wenig bekannt, was der Pathologe tut, da er nicht unmittelbar am Patienten tätig ist. Mehr als 60\% der Bevölkerung glauben, dass Pathologen lediglich verstorbene Menschen untersuchen. Die Pathologie ist jedoch eines der wenigen ganzheitlichen Fächer, deren Diagnostik den Menschen von Kopf bis Fuss erfasst und jedes andere Fachgebiet in Abklärung und Behandlung der Krankheiten unterstützt. Der Pathologe begleitet somit im Hintergrund die Krankheiten der lebenden Patienten. Die in der Pathologie erhobenen Daten sind unentbehrlich für die Epidemiologie (z. B. das Krebsregister). Eine genügend hohe Autopsierate ist wichtig für die Todesursachenstatistik und wäre ein bedeutendes Mittel der Qualitätskontrolle in der Medizin.

\section{Tätigkeit in der klinischen Pathologie}

In der modernen Medizin umfassen die Untersuchungen von Gewebe und Zellen lebender Patienten mehr als 90\% der Arbeit der Pathologen. Es werden entzündliche Krankheiten festgestellt und hinsichtlich ihrer Ätiologie klassiert. Nahezu alle Tumoren werden in der Pathologie diagnostiziert. Bei malignen Tumoren werden die therapierelevanten Parameter erfasst: Typisierung gemäss WHO-Nomenklatur, Tumorstadium, Vollständigkeit der chirurgischen Resektion, Grad des Ansprechens auf die neo-adjuvanten Chemo- und/oder Radiotherapien am chirurgischen Resektat.

Für die Behandlung maligner Tumoren gewinnt der Nachweis prädiktiver Faktoren, die den Einsatz von Medikamenten bestimmen, zunehmend an Bedeutung: Beispiele sind die Expression von Her2-Protein/Her2-Gen beim Mammakarzinom (Herceptin) oder der Nachweis von CD20 bei B-Zelllymphomen (Rituximab). Zunehmend wichtig sind die präventiven Untersuchungen in der Krebsfrüherkennung und die Therapie der Krebsvorläuferläsionen. Erwähnt seien die präventive Kolonoskopie und das Brustkrebs-Screening.

\section{Die SGPath: Ziele und Tätigkeitsfelder}

Die Schweizerische Gesellschaft für Pathologie wurde 1935 in Bern gegründet. Sie hat aktuell 368 Mitglieder. Die grosse Mehrheit ist in der klinischen Humanpathologie tätig, und ein Teil davon ist in der klinisch orientierten Forschung engagiert. Kleinere Gruppen sind in der Veterinärpathologie, in der Grundlagenforschung, Pharmaindustrie oder in klinischen Fächern aktiv. Die SGPath sowie ihre Fachgruppen Zytopathologie und Molekularpathologie haben folgende Ziele:

- Förderung des Fachs Pathologie und ihrer spezialisierten Bereiche in Lehre, Dienstleistung und Forschung.

- Kontakte zu nationalen und internationalen Vereinigungen für Pathologie.

- Durchführung der Weiter- und Fortbildung.

- Förderung der Qualitätssicherung.

- Unterstützung der Aus- und Fortbildung des technischen Personals.

Die SGPath bildet gleichzeitig die Schweizerische Sektion der Internationalen Akademie für Pathologie. Sie organisiert jährlich einen Kongress und zwei Schnittseminare. Arbeitsgruppen der SGPath widmen sich speziell der Weiter- und Fortbildung in der Pathologie einzelner Organe bzw. Organsysteme. Sie bilden den Spezialisten-Pool für fachspezifische Fragen und für die Ausarbeitung von Leitlinien der Qualitätssicherung.

\section{Problemfelder und Ausblick}

Im Rahmen der Bologna-Reformen wird die Pathologie zunehmend in Kurse integriert, in denen die Pathologie allenfalls von Vertretern anderer Fächer gelehrt würde. Wenn nun Pathologie als Grundlagenfach nicht mehr systematisch unterrichtet wird, zeichnen sich längerfristige Folgen ab: Die systematische Vermittlung der Grundlagen wird vernachlässigt. Das breitgefächerte Basiswissen künftiger Ärzte dürfte abnehmen und die Qualität der Medizin beeinträchtigen. Im Speziellen wird die Pathologie als berufliches Fach ungenügend wahrgenommen. Der bereits bestehende Mangel an Pathologen wird sich zunehmend verschärfen bei gleichzeitig steigenden diagnostischen Anforderungen. 